\title{
Contribution of Permanent-Magnet Volume Elements to No-Load Voltage in Machines
}

\author{
Maxime R. Dubois, Member, IEEE, Henk Polinder, Member, IEEE, and Jan A. Ferreira, Senior Member, IEEE
}

\begin{abstract}
In permanent-magnet (PM) electrical machines, a key role of PM material is to induce a voltage in a winding upon a relative movement of the $P M$ about that winding. Although useful, PMs are costly and machine designers should optimize the contribution of all the volume elements in PMs as much as possible. This paper presents a new mathematical expression for the no-load flux linkage generated by PMs. The mathematical expression gives the flux linkage in terms of the PM geometry. This proves to be efficient in evaluating the contribution of each local magnet volume element to generate a no-load flux linkage. We propose a method that uses the derived mathematical expression to optimize the shape of PMs, and this method is applied to the case of a conventional PM synchronous machine. The optimization process uses the results from finite-element analysis, and the resulting PM shape shows an increase of voltage induced per PM volume.
\end{abstract}

Index Terms-Cost optimization of machines, no-load voltage, permanent-magnet machines, permanent-magnet shaping.

\section{INTRODUCTION}

$\mathbf{T}$ HE step toward light and efficient electrical machines has been pushed by the use of permanent-magnet (PM) materials with a high remanent flux density. In synchronous machines, bulky rotor windings can nowadays be successfully replaced by rare-earth PM material. Although smaller in size than conventional rotor electromagnets, PMs have a much higher specific cost (in dollars per kilogram) than laminated steel and copper. Nowadays, one can purchase $\mathrm{Nd}-\mathrm{Fe}-\mathrm{B}$ magnets for about $35 \mathrm{US} \$ / \mathrm{kg}$, where the cost for standard $0.5-\mathrm{mm}$-thick steel laminations is around $1.5 \mathrm{US} \$ / \mathrm{kg}$. In many cases, the cost of PM material is a significant part of the total machine costs.

The subject of PM shapes has been discussed in [1]-[3]. However, in those contributions, the PM shapes are not optimized with respect to the amount of induced voltage per cubic millimeter of PM material. Do all volume elements $d v$ inside the PMs contribute equally to induce a no-load voltage in the stator winding? Is it possible that some part of the PM volume brings a high contribution to the no-load voltage, while other parts would bring a lower contribution? In this paper, the aim is to find a general method to minimize the cost of PMs in a given machine, by minimizing the PM volume in that machine. This is done by

\footnotetext{
Manuscript received June 21, 2001; revised January 24, 2003. This work was supported in part by Delft University of Technology and in part by the FCAR, Quebec, Canada.

The authors are with the Delft University of Technology, 2628 CD Delft, The Netherlands (e-mail: m.dubois@its.tudelft.nl; h.polinder@its.tudelft.nl; j.a.ferreira@its.tudelft.nl).

Digital Object Identifier 10.1109/TMAG.2003.809874
}

identifying which parts of the PM volume have a high contribution to the total magnetic flux linkage induced in the stator winding at no-load.

In order to obtain the local PM contribution to the total flux linkage, a mathematical expression is derived for the flux linkage generated by a PM. This expression integrates a function over the sole volume of all the PMs considered in the machine. Usually, the flux linking a coil is obtained by computing the flux density over the coil surface. In this paper, the no-load flux linkage is obtained by calculating field values over the PM geometry rather than the coil geometry. For the optimization of PM shapes, this new way of expressing flux linkage appears to be more practical than the conventional expression. Using this mathematical expression, we can attribute to each volume element $d v$ inside the PM a local contribution to the no-load flux linkage.

In the end, the machine designer is more interested in the induced voltage, rather than flux linkage. According to Faraday's law, the induced voltage is obtained when the no-load flux linkage varies with time. In PM machines, this is obtained by moving the PMs with a given speed. With a case example, we show that the contribution to no-load flux linkage for a given PM volume element $d v$ varies with the rotor position. Therefore, its contribution to the instantaneous no-load voltage also varies with position and time. However, a contribution to the average no-load voltage can be obtained by integrating the no-load voltage over all electrical angles during one half-cycle. In that case, each volume element inside the PMs has a fixed contribution.

The last part of the paper presents a method used to create PM shapes so as to maximize the average no-load voltage per volume of PM material. This method uses the theoretical results proposed in the paper. With the use of finite-element analysis (FEA), we show that such a method allows the design of costeffective PM shapes. The case example given in this paper is a conventional PM synchronous machine. With FEA results, we show that $\mathrm{V}$-shaped magnet blocks have a higher ratio of $\mathrm{V} / \mathrm{mm}^{3}$ than conventional rectangular blocks.

\section{PM LocAl CONTRIBUTION TO FLUX LiNKAGE}

\section{A. Problem Definition, General Case, and Assumptions}

In Section II-B, a mathematical expression will be derived for the no-load flux linkage generated by the PMs. The derivation is meant to be general and can be applied to different kinds of PM machines. A generalized PM machine is shown in Fig. 1, where the shapes of iron, PM, and coil inside the machine boundaries are arbitrary. 


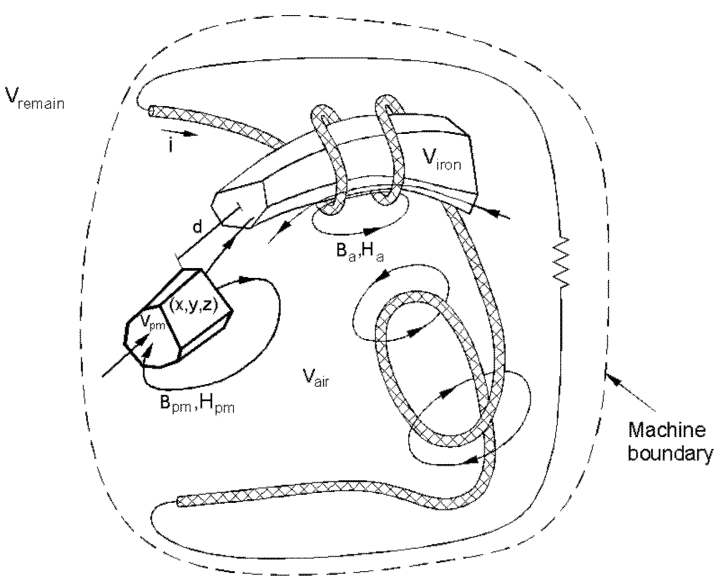

Fig. 1. Generalized PM machine, with arbitrary iron, PM, and coil shapes.

In Fig. 1, all PM material in the machine is contained in the volume $V_{\text {pm }}$, all steel is contained inside the volume $V_{\text {iron }}$, and the remaining space within the machine boundary is contained inside the volume $V_{\text {air }}$. Outside the machine boundary, the vector quantities $\boldsymbol{B}_{a}, \boldsymbol{B}_{\mathrm{pm}}, \boldsymbol{H}_{a}$, and $\boldsymbol{H}_{\mathrm{pm}}$ created by the coil and the PMs are negligible. Also, the PM and coils possibly existing outside the machine create a magnetic field that is negligible inside the machine boundary. In itself, the machine boundary is defined as a region in space containing all the magnetic fields $\boldsymbol{H}_{a}$ and $\boldsymbol{H}_{\mathrm{pm}}$ formed by the machine coil and the machine PM and inside of which the externally created magnetic fields are negligible.

The first step in the elaboration of a shaping method for PM blocks is the derivation of an expression for the no-load flux linkage. Before going through the details of this mathematical derivation, three assumptions are stated.

1) Assumption of Rigid Magnetization for PMs: All PMs inside the machine boundary have rigid magnetization, i.e., $\boldsymbol{B}(\boldsymbol{H})$ curves inside the PM are defined by (1)

$$
\vec{B}=\mu_{0} \vec{H}+\vec{B}_{r}
$$

In (1), $\boldsymbol{B}$ and $\boldsymbol{H}$ are, respectively, the flux density and magnetic field intensity inside the PMs. $\boldsymbol{B}_{r}$ is the PM remanent flux density and has a constant magnitude. The PM recoil permeability $\mu_{\text {rec }}$ is assumed to be 1 . These assumptions are not serious limitations to the derivation exposed further if the permanent magnets like $\mathrm{Nd}-\mathrm{Fe}-\mathrm{B}$ magnets are used. "Real-life" $\mathrm{Nd}-\mathrm{Fe}-\mathrm{B}$ magnets have typical values of their recoil relative permeability in the area of 1.0 to 1.1 and have a $\boldsymbol{B}(\boldsymbol{H})$ characteristic that is approximately linear from $B=B_{r}$ down to $B=0 \mathrm{~T}$.

As mentioned in the last paragraph, $\boldsymbol{B}_{\boldsymbol{r}}$ is assumed to have a constant magnitude for any point inside the PM, no matter the intensity of the magnetic field $\boldsymbol{H}$ at that location. However, the orientation of the vector $\boldsymbol{B}_{r}$ may vary inside the PM. In this paper, we consider an example where the remanent flux density has constant magnitude and orientation throughout the volume of a PM. However, a companion paper [4] describes the advantages of varying the orientation of $\boldsymbol{B}_{r}$ inside the PM volume.
2) Assumption of Ideal Ferromagnetic Material for Steel: It is assumed that all iron parts are ideal: they have infinite permeability and are nonsaturable. Standard "real-life" cold-rolled steel materials have an initial relative magnetic permeability many orders of magnitude higher than unity. As a consequence, this assumption of ideal iron appears to be reasonable. However, the validity of the method proposed in this paper needs to be carefully analyzed for cases where iron saturation would occur. This is not done in the paper.

3) Assumption of Constant Magnetic Vector Potential A Throughout the Conductors' Cross Section: The magnetic vector potential $\boldsymbol{A}$ is assumed to have constant magnitude and direction through any given cross section of the conductors. The word "conductors" here only refer to the conductors forming the coils inside the machine boundary. This is the theoretical case of filamentary conductors, which have currents flowing through an infinitely small cross section.

In practice, the variation of the magnetic vector potential $\boldsymbol{A}$ is very small when the conductors are wound around a ferromagnetic core, as in most electrical machines. In the latter case, most of the magnetic flux flows inside the iron and the variation of $\boldsymbol{A}$ through the conductor cross section may be considered as negligible compared to the absolute value of $A$ on that conductor.

For conductors in empty space (or air), the variation of $\boldsymbol{A}$ through the conductor cross section may be considered as negligible if the conductor cross section is made sufficiently small.

\section{B. Mathematical Derivation}

In the system of Fig. 1, we consider a coil made of several turns, where a current $i$ is allowed to flow. We write the product $i \lambda$ "seen" by that coil

$$
i \lambda=\left[\iint_{S_{\text {cond }}} \vec{j} \cdot d \vec{s}_{\text {cond }}\right]\left[\int_{S_{\text {coil }}} \int_{B} \cdot d \vec{s}_{\text {coil }}\right]
$$

where $\lambda$ and $B$ are the flux linkage and flux density linking the coil, including the flux created by the PM and the flux created by current $i . j$ is the current density vector inside the conductor, $S_{\text {coil }}$ is a surface bounded by the complete coil, and $S_{\text {cond }}$ is the cross section of the conductor, as shown in Fig. 2. It should be noted that the boundary of $\mathrm{S}_{\text {coil }}$ can be set anywhere inside the conductor, as long as the assumption of constant A inside the conductor is met (assumption 3 ).

As discussed by [5], $i \lambda$ is the sum of the magnetic energy and magnetic coenergy "seen" by the coil. It may look like an unusual starting point, but it will be useful later in this derivation.

In (2), we replace $B$ by the curl of the magnetic vector potential $\boldsymbol{A}$, and Stoke's theorem is applied to obtain

$$
i \lambda=\left[\iint_{S_{\text {cond }}} \vec{j} \cdot d \vec{s}_{\text {cond }}\right]\left[\oint_{l_{\text {coil }}} \vec{A} \cdot d \vec{l}_{\text {coil }}\right] .
$$

As described in [6], the conductor can be divided into an infinite number of small current tubes carrying infinitesimal currents $d i$ and inside of which the current density $\boldsymbol{j}$ is conservative (electric flux density $\boldsymbol{D}$ varies very slowly with time). Inside each infinitesimally small current tube, the current density $\boldsymbol{j}$ is 


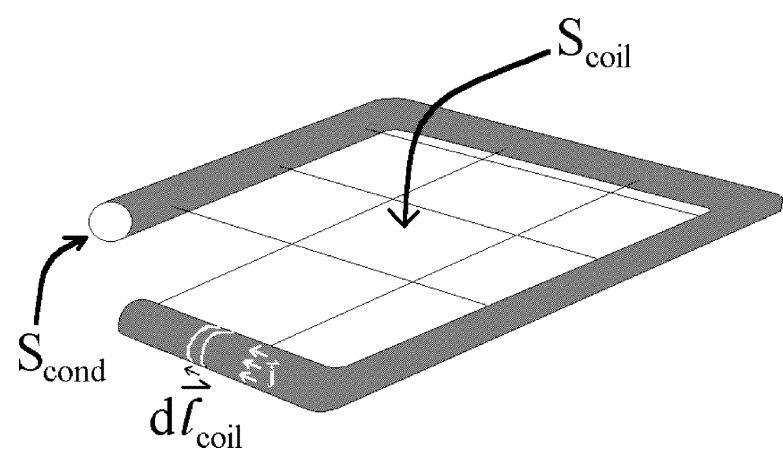

Fig. 2. $S_{\text {coil }}$ and $S_{\text {cond }}$ for one turn of the coil.

parallel to $d \boldsymbol{l}_{\text {coil }}$ and is also parallel to $d \boldsymbol{s}_{\text {cond }}$. Then, (3) can be written as a volume integral

$$
i \lambda=\iint_{V_{\text {cond }}} \int \vec{j} \cdot \vec{A} d v
$$

if the variation of $\boldsymbol{A}$ throughout the conductor cross section is negligible (assumption 3). In (4), $V_{\text {cond }}$ is the total volume of conducting material forming the coil. Equation (4) can be expressed in terms of $\boldsymbol{B}$ and $\boldsymbol{H}$, as demonstrated in Appendix I. We rewrite (4) as

$$
i \lambda=\iint_{V_{\text {universe }}} \vec{B} \cdot \vec{H} d v .
$$

The development of (4) and (5) is done in the same way here as for the expression of magnetostatic energy derived in common textbooks [6]-[8]. However, (4) and (5) are not magnetostatic energy, but rather the sum of magnetostatic energy and coenergy.

The three variables $\lambda, \boldsymbol{H}$, and $\boldsymbol{B}$ of (5) have a component created by the current in the coil and a component created by the PMs. The assumptions of linear PM (assumption 1) and ideal iron (assumption 2) allow us to subdivide $\lambda, \boldsymbol{H}$, and $\boldsymbol{B}$ into $\lambda_{a}, \boldsymbol{H}_{a}, \boldsymbol{B}_{a}$ (component created by the current flowing in the coil, when $B_{r}=0 \mathrm{~T}$ ), and $\lambda_{\mathrm{PM}}, \boldsymbol{H}_{\mathrm{PM}}$, and $\boldsymbol{B}_{\mathrm{PM}}$ (component created by the PM alone, when $i=0$ ). The components $\boldsymbol{H}_{a}$, $\boldsymbol{B}_{a}, \boldsymbol{H}_{\mathrm{PM}}$, and $\boldsymbol{B}_{\mathrm{PM}}$ are shown in Fig. 1. We rewrite (5) as

$$
\left.i\left(\lambda_{a}+\lambda_{\mathrm{PM}}\right)=\iint_{V_{\text {universe }}} \int_{\vec{B}_{a}}+\vec{B}_{\mathrm{PM}}\right) \cdot\left(\vec{H}_{a}+\vec{H}_{\mathrm{PM}}\right) d v .
$$

It is shown in Appendix II how the product $i \lambda_{\mathrm{PM}}$ can be written in terms of an integral over the PM volume. With the result of Appendix II, we rewrite (6) as

$$
i \lambda_{\mathrm{PM}}=\iint_{V_{\mathrm{PM}}} \int_{H_{a}} \cdot \vec{B}_{r} d v
$$

where $\lambda_{\mathrm{PM}}$ is the no-load flux linking the coil, and $\boldsymbol{B}_{r}$ is the remanent flux density vector of the PM. From (7), we obtain an expression for $\lambda_{\mathrm{PM}}$

$$
\lambda_{\mathrm{PM}}=\iint_{V_{\mathrm{PM}}} \int\left[\frac{\vec{H}_{a}}{i}\right] \cdot \vec{B}_{r} d v .
$$

Equation (8) expresses the flux linkage in terms of a function, which is integrated over the sole volume of all the PMs interacting with the coil. It gives every volume element $d v$ inside the PMs a contribution to the total flux linking the coil at no-load.

For any rotor position, one may inject a current $i$ in the coil and compute $\boldsymbol{H}_{a}$ in the volume of the PM. Integrating the dot product of $\boldsymbol{H}_{a}$ with $\boldsymbol{B}_{r}$ over the PM volume gives the no-load flux linking the coil into which the current was injected.

There is a reciprocity relationship between (8) and the conventional expression of no-load flux linkage given by (9).

$$
\lambda_{\mathrm{PM}}=\iint_{S_{\mathrm{coil}}} \vec{B}_{\mathrm{PM}} \cdot d \vec{s}_{\mathrm{coil}}
$$

In (9), the flux linkage at no-load is defined as a magnetic quantity created by the PM $\left(\boldsymbol{B}_{\mathrm{PM}}\right)$ acting on the geometry of the coil $\left(S_{\text {coil }}\right)$, while in $(8), \lambda_{\mathrm{PM}}$ is expressed as a magnetic quantity created by the coil $\left(\boldsymbol{H}_{a}\right)$ and acting on the geometry of the $\mathrm{PM}\left(V_{\mathrm{PM}}\right)$.

\section{PM LOCAL CONTRIBUTION TO INSTANTANEOUS AND AVERAGE No-LOAD VOLTAGE}

\section{A. Instantaneous No-Load Voltage}

From (8), we may define an expression for the local contribution $c(x, y, z)$ of each PM volume element to the total no-load flux linking the coil

$$
c(x, y, z)=\left[\frac{\vec{H}_{a}(x, y, z)}{i}\right] \cdot \vec{B}_{r}(x, y, z) .
$$

It must be noted that $c(x, y, z)$ is not written as a function of the current $i$, because for a linear system, $\boldsymbol{H}_{a}$ will be proportional to $i$. Modifying $i$ will not affect $c(x, y, z)$.

Equation (10) gives each volume element inside the PM a contribution to the no-load flux linkage. However, if (10) is used to calculate the contribution of each PM volume element to the no-load voltage $e$, the time-dependence of $c$ must be carefully analyzed. From Faraday's law, we know that the no-load voltage is time-dependent and in fact results from the motion of all these PM volume elements. Equation (10) is written as a time-independent expression, which is not satisfactory when it comes to the calculation of $e$. In this section, we will analyze the role played by time and motion in the contribution of each PM volume element to the no-load voltage.

We set an arbitrary orthogonal coordinate system $(x, y, z)$, which is attached to the PMs. A distance $d$ exists between the origin of this coordinate system $(x, y, z)$ and the fixed stator. For the moment, we will assume $d$ to be constant in time, and we will only allow the current to vary in time. Let us write $c$ as a function of the coordinate system $(x, y, z)$ and the distance $d$

$$
\begin{aligned}
& c\left(x, y, z, d_{x}, d_{y}, d_{z}, t\right) \\
& =\left[\frac{\vec{H}_{a}\left(x-d_{x}, y-d_{y}, z-d_{z}, t\right)}{i(t)}\right] \cdot \vec{B}_{r}(x, y, z)
\end{aligned}
$$

where

$$
d=\sqrt{d_{x}^{2}+d_{y}^{2}+d_{z}^{2}} .
$$




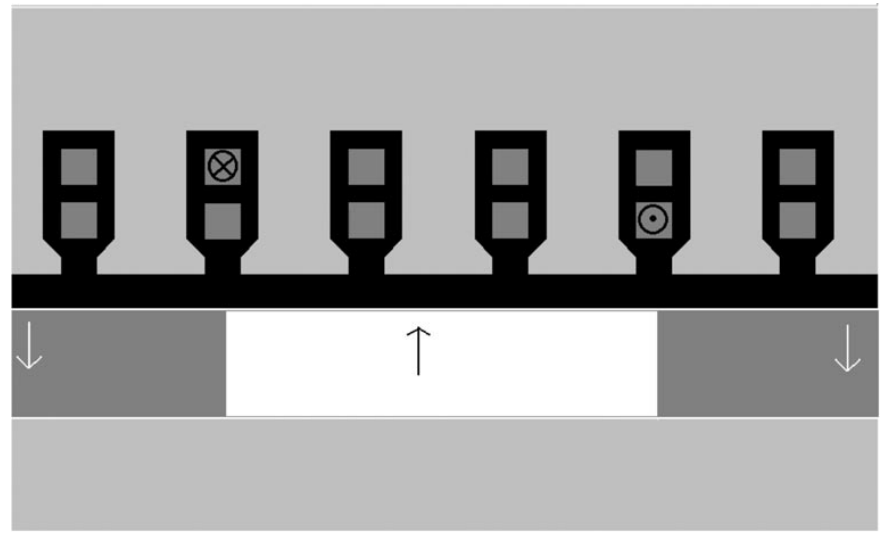

Fig. 3. Stator and rotor of a three-phase conventional PM synchronous machine. Only phase A is fed as shown here. Rotor position for $t=\mathrm{t}_{1}$.

In (11), it must be noted that $B_{r}$ is expressed as a function of $(x, y, z)$ only and is independent of time $t$ and distance $d$. However, $\boldsymbol{H}_{a}$ is expressed as a function of the distance $d$, because its reference is not attached to the PM coordinate system.

If we allow the distance $d$ between the PM and the stator to vary with time, we can rewrite (12) as

$$
\begin{aligned}
& c(x, y, z, t) \\
& \quad=\left[\frac{\vec{H}_{a}\left(x-d_{x}(t), y-d_{y}(t), z-d_{z}(t), t\right)}{i(t)}\right] \cdot \vec{B}_{r}(x, y, z) .
\end{aligned}
$$

Clearly, as soon as the PM is moving and $d$ becomes a function of time, the local contribution function $c$ is not fixed in time. The instantaneous no-load voltage may be written as

$$
e=\frac{d \lambda_{\mathrm{PM}}}{d t}=\iiint_{V_{\mathrm{PM}}} \frac{\partial c(x, y, z, t)}{\partial t} d v
$$

where $c(x, y, z, t)$ is defined by (13).

Until now, this discussion was general. Equations (8) and (14) can be used to calculate the no-load flux linkage $\lambda_{\mathrm{PM}}$ and voltage $e$ for virtually any magnetic circuit containing PM material, as long as the three assumptions of Section II-A are met. For the rest of the paper, a real machine example is used. The machine geometry used is a conventional PM synchronous machine. This example is shown in Fig. 3. The machine shown in Fig. 3 has one slot per pole per phase and its design specifications are given in Appendix III. If we impose a movement in the $x$ axis with a fixed speed $\kappa$ to the rotor, we have

$$
\begin{aligned}
d_{x}(t) & =\kappa t \\
d_{y}(t) & =k_{y} \\
d_{z}(t) & =k_{z}
\end{aligned}
$$

where $k_{y}$ and $k_{z}$ are constants. Fig. 4(a) and (b) illustrates the local contributions of two points inside the PM at two different positions of the rotor. In both rotor positions, the coordinates $(x, y, z)$ of these two points stay the same. In Fig. 4, only one coil is fed with a current $i$ in order to obtain the contribution to the flux linkage in that coil. This explains the nonuniform distribution of $c$ inside adjacent magnets.

In Fig. 4(a), the left point has a higher absolute contribution than the point in the middle of the PM, where in Fig. 4(b), the

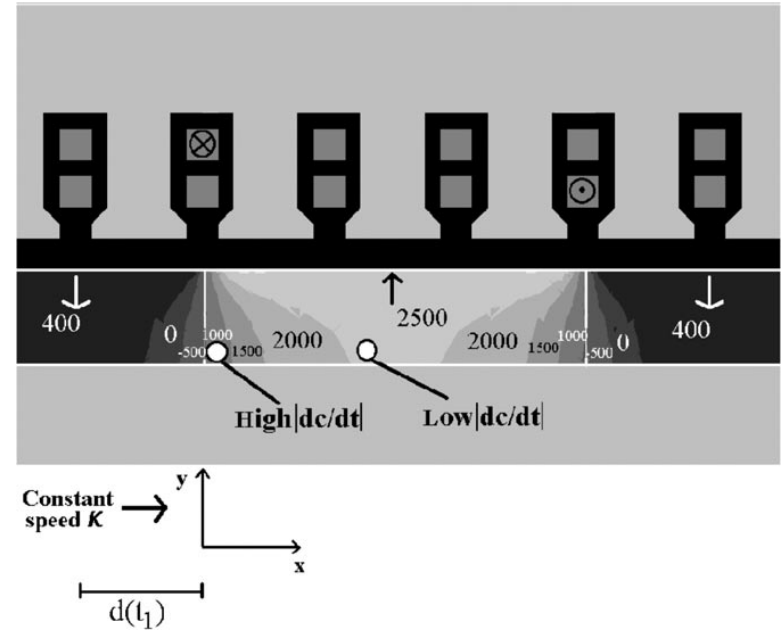

(a)

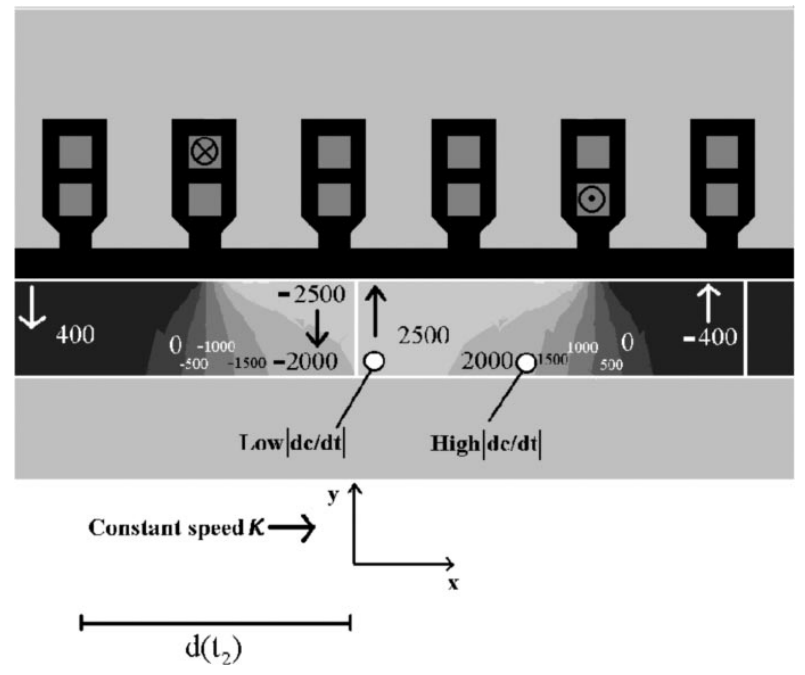

(b)

Fig. 4. (a) Dot product of $\boldsymbol{H}_{a} \cdot \boldsymbol{B}_{r}$ computed with FEA for $d\left(t_{1}\right)=\kappa t_{1}$, and $t=t_{1}$. Two points are indicated, showing high and low contribution to no-load voltage. $\boldsymbol{B}_{r}$ is constant and homogenous in the volume of each PM. The coil current is $i=10 \mathrm{~A}$. (b) Dot product of $\boldsymbol{H}_{a} \cdot \boldsymbol{B}_{r}$ computed with FEA for $d\left(t_{2}\right)=\kappa t_{2}$, and $t=t_{2}$. The same two points as in (a) are indicated inside the PM. The contribution to the no-load voltage is different. $\boldsymbol{B}_{r}$ is constant and homogenous in the volume of each PM. The coil current is $i=10 \mathrm{~A}$.

same left point has a lower absolute contribution than the middle point.

From (13) and (14), we conclude that the contribution of each PM volume element to the no-load voltage is time-dependent, and from the example of Fig. 4, we conclude that this contribution is constantly changing inside the volume of the PM while the rotor is moving. At some location inside the PM, the contribution to the no-load voltage changes from high to low upon the rotor translation, while at another location, it changes from low to high.

The first objective of this paper is to evaluate how we can remove the volume elements having the lowest contribution to the no-load voltage. Since the shape of the PM cannot be modified during its motion, the knowledge of the instantaneous local contribution to $e$ has little practical interest, because it is changing constantly with the motion of the rotor. 


\section{B. Average No-Load Voltage}

It is more practical to obtain the local contributions of PM elements to the average no-load voltage $E_{\text {ave }}$, because the average no-load voltage is time-independent. Over one electrical period, the average no-load voltage is of course zero for a symmetrical machine. However, it is possible to define the average no-load voltage over one half-period

$$
E_{\text {ave }}=\frac{2}{T} \int_{t_{1}}^{t_{1}+\frac{T}{2}} \frac{d \lambda_{\mathrm{PM}}}{d t} d t=\frac{2}{T} \int_{\lambda_{\mathrm{PM}}\left(t_{1}\right)}^{\lambda_{\mathrm{PM}}\left(t_{1}+\frac{T}{2}\right)} d \lambda_{\mathrm{PM}}
$$

where $E_{\text {ave }}$ is the average no-load voltage over one half-period, and $T$ is the electrical period. In (16), the integration boundary $t=t_{1}$ is the time instant where the rotor PM is completely facing the stator pole, and for which the no-load flux linkage is maximum [see Fig. 4(a)]. The time instant $t=t_{1}+T / 2$ is the time instant for which the next PM is completely facing the stator pole and for which the flux linkage has the same value as for $t=t_{1}$, but with opposite polarity. From (16), we can write

$$
E_{\text {ave }}=-\frac{4}{T} \lambda_{\mathrm{PM}}\left(t_{1}\right)
$$

Inserting the result obtained with (8) into (17), we obtain

$$
E_{\text {ave }}=-\frac{4}{T i\left(t_{1}\right)} \iint_{V_{\mathrm{PM}}} \int \vec{H}_{a}\left(t_{1}\right) \cdot \vec{B}_{r} d v .
$$

In (18), only one sample of $i$ and one pattern of magnetic field intensity $\boldsymbol{H}_{a}$ are used, that is, for $t=t_{1}$. In this case, each volume element $d v$ has a fixed contribution.

In (18), only one value of $i$ is used. However, it is not required that the injected current $i$ remains constant during the rotor displacement over one half-cycle. In reality, the no-load voltage is not a function of the current waveform. Even though the no-load voltage cannot be measured when current is flowing in the coil, the average no-load voltage is still present, and the proposed method allows the calculation of its value under loaded conditions. It must be noted that setting $i=0$ in (18) would give an indetermination, because then $i=0$ and $\boldsymbol{H}_{a}=0$.

\section{METHOD FOR THE SHAPING OF PM}

The mathematical expression given by (18) allows us to propose a method for the optimal shaping of PM. This method is used to shape the PMs, so as to obtain an increased average no-load voltage across one coil of a machine per cubic millimeter of PM material. The method may use either FEA or analytical calculation in the following manner.

- Consider the rotor position for which the PM faces the stator pole completely, as in Fig. 4(a).

- Replace all PM by vacuum in the FEA model, or in the analytical calculation.

- Inject a current $i=\mathrm{I}$ in the coil across which we want to obtain the average no-load voltage.

- All other coils have $i=0$.

- Calculate the field $\boldsymbol{H}_{a}$ inside the volume that would be filled by all PM.

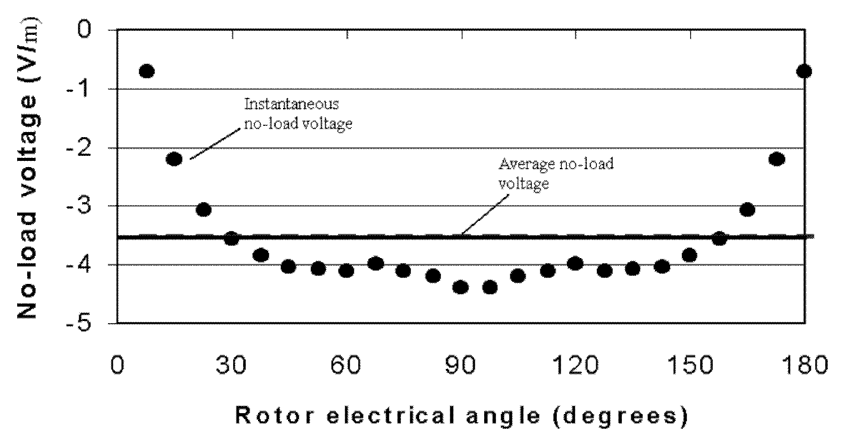

Fig. 5. Instantaneous and average no-load voltage computed point-by-point with FEA, for every rotor position. The PM shape type has adjacent rectangular blocks (same as Fig. 3). The result is given in volts per meter because 2-D FEA software is used.

- Do the dot product of $\boldsymbol{H}_{a}$ with $\boldsymbol{B}_{r}$ inside all the PM volume in the machine. This will give out the contribution of each volume element inside the PM.

- Remove the volume elements with the lowest values of $\boldsymbol{H}_{a} \cdot \boldsymbol{B}_{r}$.

\section{APPLICATION: FEA Results}

In this section, the proposed method is used to optimize the shape of PM in the conventional PM synchronous machine of Fig. 3. In Fig. 3, the distance between two adjacent PMs is zero. It is common practice to keep a certain distance between two adjacent PMs, where it is expected that a strong flux leakage between magnets is found. In the area near the boundary between two magnets, the flux created by the PMs does not contribute significantly to the flux in the stator coil. Machine designers consider the frontier area of two PMs as less useful PM material and, therefore, they tend to remove PM material in that region. The obtained magnet usually has a rectangular shape, with a distance of 0.1 to 0.3 times the pole pitch between adjacent magnets. In this paper, we give ourselves more freedom in the types of PM shapes than only rectangular shapes.

Before going through the PM shaping method, the average no-load voltage and the no-load instantaneous voltages are obtained by moving the rotor of Fig. 3 about the winding. For every position, the no-load voltage is computed with FEA. The resulting waveform is shown in Fig. 5. We will use this result to validate the method proposed in Section IV.

With the new method proposed in Section IV, all PMs of Fig. 3 are replaced by vacuum. A current $i=10 \mathrm{~A}$ is injected in the coil. The dot product of $\boldsymbol{H}_{a}$ and $\boldsymbol{B}_{r}$ is computed inside the volume of the PM by using 2-D FEA software. The distribution of $\boldsymbol{H}_{a} \cdot \boldsymbol{B}_{r}$ is shown inside the PM in Fig. 4(a), which represents the contribution of each PM volume element $d v$ to the no-load flux linkage $\lambda_{\mathrm{PM}}$ in the coil, and to the average no-load voltage $E_{\text {ave }}$ across that coil.

It must be noted that in this example, the current is injected in only one coil of the machine. As a result, the magnetic field $\boldsymbol{H}_{a}$ is spread throughout all the PMs in the machine, and the volume integration must take place over all PMs. Usually, windings and rotor poles are identical for all poles throughout the machine. Thus, volume integration can take place on only one pole due to the symmetry between poles. In a symmetrical distributed 


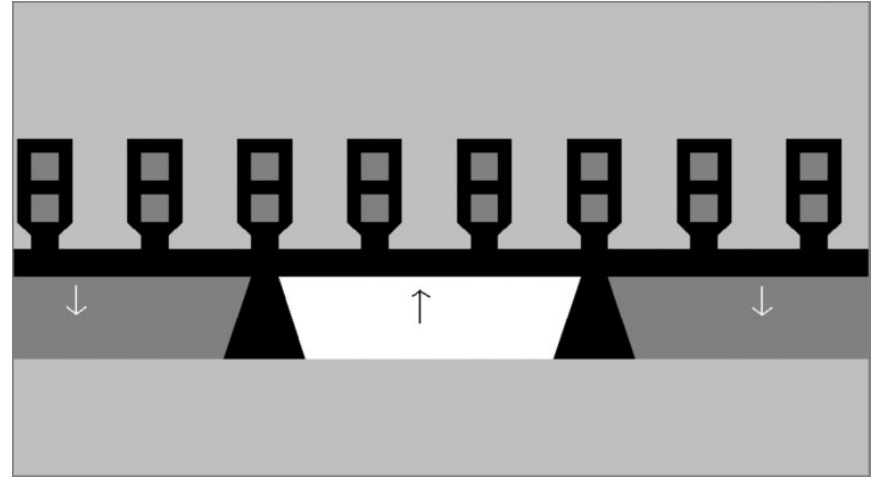

Fig. 6. New PM shapes obtained with the method.

winding, for example, it may be more useful to feed a complete winding, instead of individual coils. Even though this is an easy step to make, the symmetrical case is not covered in the paper, which aims to be more general.

As expected, the region in Fig. 4(a) near the boundary between two PMs has the lowest contribution to the average no-load voltage. If PM elements are to be removed from the PM volume, they should indeed be taken from that region near the frontier between neighbor magnets. However, Fig. 4(a) suggests that PMs should not be cut in rectangles, as is usually done, but rather with a V-shape. The PM could be shaped as shown in Fig. 6.

In Fig. 6, the section of PM material with the new V-shape has a cross section of $120 \mathrm{~mm}^{2}$ per magnet, compared with $144 \mathrm{~mm}^{2}$ per magnet for the initial block shape of Fig. 3. The no-load flux linkage for the new $\mathrm{V}$-shape in the rotor position shown in Fig. 6 is $0.0160 \mathrm{~Wb} / \mathrm{m}$, compared with $0.0176 \mathrm{~Wb} / \mathrm{m}$ with the initial block shape. The decrease in PM cross section is $17 \%$, while the decrease in flux linkage and average no-load voltage is only $9 \%$. The amount of $\mathrm{V} / \mathrm{mm}^{3}$ generated by the PM at no-load has increased by $8 \%$ with the new V-shape. Table I shows the no-load flux linkage and average no-load voltage for the two PM shapes.

Columns 3 and 5 in Table I are computed using the method shown in the paper with (8) and (18). For comparison purposes, column 2 gives the no-load flux linkage obtained by integrating $\boldsymbol{B}_{\mathrm{PM}}$ over the coil area [conventional method expressed by (9)]. The difference between the conventional way of computing the no-load flux linkage (column 2) and the expression derived in the paper (column 3 ) is below $0.6 \%$ if the relative recoil permeability of the $\mathrm{Nd}-\mathrm{Fe}-\mathrm{B}$ material is unity.

We can expect the difference in flux linkage between the conventional and the new calculation method to grow as the relative recoil permeability moves away from unity. A common value of $\mu_{\mathrm{rec}}=1.09$ has also been used with the conventional expression (column 2), and the difference between column 2 and column 3 has increased to about 4\% (see Table I).

The average no-load voltage obtained with the proposed method (column 5) is also closely corroborated by the point-by-point estimation (column 4). For the sake of completeness, the average and instantaneous voltages are also computed with FEA by moving the rotor point-by-point with the V-shape PM. The result is illustrated in Fig. 7.
TABLE I

FEA RESULTS FOR TWO DIFFERENT PM SHAPES

\begin{tabular}{|c|c|c|c|c|c|}
\hline $\begin{array}{l}\text { PM } \\
\text { Shape }\end{array}$ & 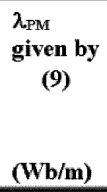 & $\begin{array}{l}\begin{array}{l}\lambda_{\mathrm{PM}} \\
\text { given by } \\
\quad(8)\end{array} \\
(\mathrm{Wb} / \mathbf{m}) \\
\end{array}$ & $\begin{array}{l}\text { Eave }_{\text {point by }} \\
\text { point } \\
\text { with } \\
\text { FEA } \\
(\mathrm{V} / \mathrm{m})\end{array}$ & $\begin{array}{c}\mathbf{E}_{\text {are }} \text { given by } \\
\text { (18) }\end{array}$ & $\left(\mathrm{V} / \mathrm{mm}^{3}\right)$ \\
\hline $\begin{array}{l}\text { Square } \\
\text { PM } \\
\text { (fig. 3) }\end{array}$ & 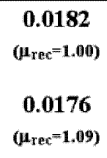 & 0.0183 & $\begin{array}{c}-3.64 \\
\left(\mu_{\mathrm{rec}}=1.00\right) \\
-3.53 \\
\left(\mu_{\mathrm{rec}}=1.09\right)\end{array}$ & -3.66 & $\begin{array}{c}0.0254 \\
\left(a_{\mathrm{rec}}=1.09\right)\end{array}$ \\
\hline $\begin{array}{l}\text { V-shape } \\
\text { PM } \\
\text { (fig. 6) }\end{array}$ & $\begin{array}{c}0.0165 \\
\left(\mu_{\mathrm{rec}}=1.00\right) \\
\\
0.0160 \\
\left(\mu_{\mathrm{rec}}=1.09\right)\end{array}$ & 0.0166 & $\begin{array}{c}-3.30 \\
\left(\mu_{\mathrm{rec}}=\mathbf{1 . 0 0}\right) \\
-\mathbf{3 . 2 0} \\
\left(\mu_{\mathrm{rec}}=1.09\right)\end{array}$ & -3.33 & $\begin{array}{c}0.0278 \\
\left(\mu_{\mathrm{rec}}=1.09\right)\end{array}$ \\
\hline
\end{tabular}

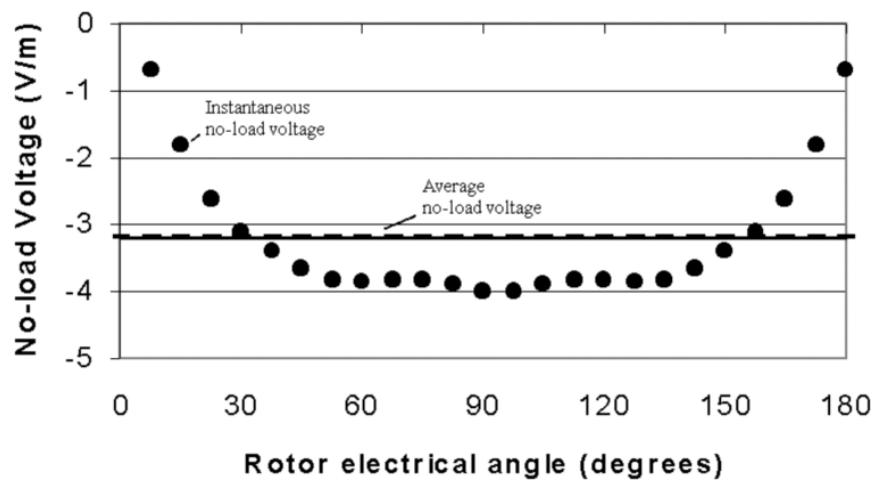

Fig. 7. Instantaneous and average no-load voltage computed for every rotor position, using FEA. PM shape type has V-shape (Fig. 6).

The induced voltage of Fig. 7 displays a more sinusoidal waveform than that of Fig. 5. The average no-load voltage is matched by the value obtained with the proposed method within $4 \%$. Although small, this $4 \%$ difference is once again explained by the assumption in the proposed method, of PM material having unity recoil relative permeability. In the FEA model used to compute the flux linkage with the point-by-point calculation, real material parameters have been used $\left(\mu_{\text {rec }}=1.09\right)$.

In this example, the optimization method gives an improvement of $8 \%$ in the amount of volts per cubic millimeter of PM material. With the expression of the no-load flux linkage given by (8), we note that the possible improvements by magnet shaping are very much dependent upon the fringing of the magnetic field in the interpole area.

A thin air gap and a large pole pitch will result in very little possible improvement of the $\mathrm{V} / \mathrm{mm}^{3}$. This is due to the presence of a largely one-dimensional magnetic field, which according to (8) gives a homogenous contribution for the PM volume elements. However, a thick air gap and a short pole pitch will see a large part of the magnetic field created by the stator winding fringing the PM border. In such cases, the shape optimization process described in this paper may bring good PM material savings. This is especially the case in surface magnet transverse-flux PM machines, where pole pitches of $1 \mathrm{~cm}$ have been reported [9]. This is the subject of a companion paper [4]. 


\section{CONCLUSION}

The paper proposes a new method to maximize the amount of $\mathrm{Wb} / \mathrm{mm}^{3}$ and $\mathrm{V} / \mathrm{mm}^{3}$ of PM material in a PM machine. The method is based on a new mathematical expression for the flux linkage created by a PM, which is derived in the paper. The expression describes the no-load flux linkage as the interaction of the stator-created magnetic field with the PM remanent flux density within the PM volume. The flux linkage is calculated by injecting a current in the winding considered, while the PM volume is replaced by vacuum. A plot of the contribution to the no-load flux linkage can be drawn inside the PM, and the areas of lower contributions may be left out.

Since the local contribution of each PM volume element to the instantaneous no-load voltage varies when the rotor moves about the winding, we chose to work with the contribution to the average no-load voltage, which is time-independent.

This method was applied to a configuration of a three-phase conventional PM synchronous machine, with a pole pitch of $24 \mathrm{~mm}$. The new expressions of no-load flux linkage and average voltage derived in the paper are in good agreement with the conventional formulas. The no-load flux linkage and average no-load voltage are within $0.6 \%$ of the values calculated with the standard mathematical expressions for PM with $\mu_{\text {rec }}=1.00$. The difference between the two methods was below $4 \%$ when real PM material properties were used $\left(\mu_{\mathrm{rec}}=1.09\right)$.

After applying this optimization method, the optimized PM shape had a V-shape rather than a rectangular shape. The average no-load voltage per cubic millimeter was increased by $8 \%$. This improvement can be higher in the case of higher ratio of air gap over pole pitch, as it could be the case for a transverse-flux PM machine with surface magnets.

\section{APPENDIX I}

In (4), $V_{\text {cond }}$ is the total volume of conducting material in one coil inside the machine. We must remind the reader that the machine stator is assumed as composed of several coils, which may or may not be symmetrically laid out in the stator. Each coil is made of several turns, and we are interested in obtaining the flux linkage of one coil in order to determine the no-load voltage across that coil. Because we are interested only by the flux linking that one coil, all other coils are considered in the present analysis as having no current flowing through them. All other free charges in the volume $V_{\text {remain }}$ are also assumed to stand still. Let us also imagine that the only PM bodies found in the universe are contained inside the machine boundary. The presence or the absence of a current density $\boldsymbol{j}$ and PM bodies outside the machine boundary will not affect the value for flux linkage $\lambda$ that we will obtain in the coil, provided that the machine boundary is sufficiently large. As a consequence, the assumption of zero current density $\boldsymbol{j}$ and no PM in the entire universe extending outside the machine boundary can be made.

We can rewrite (4) by extending the integration boundary to all universe, given the current density $j$ is zero everywhere, except in the coil for which we want to calculate the no-load flux linkage

$$
i \lambda=\iint_{V_{\text {universe }}} \int \vec{j} \cdot \vec{A} d v
$$

With the assumption of conservative $\boldsymbol{j}$ (electric flux density $D$ varies very slowly with time), we rewrite (AI.1)

$$
i \lambda=\iint_{V_{\text {universe }}}(\nabla \times \vec{H}) \cdot \vec{A} d v
$$

Using a well-known vector identity (see [10]), we obtain

$$
i \lambda=\iint_{V_{\text {universe }}} \nabla \cdot(\vec{H} \times \vec{A}) d v+\iint_{V_{\text {universe }}} \int \vec{H} \cdot(\nabla \times \vec{A}) d v .
$$

We obtain (AI.4) by replacing the curl of $\boldsymbol{A}$ by $\boldsymbol{B}$ in the second term, and by applying Stoke's theorem to the first term

$$
i \lambda=\oiint_{S_{\text {universe }}}(\vec{H} \times \vec{A}) \cdot \vec{n} d s+\iint_{V_{\text {universe }}} \vec{H} \cdot \vec{B} d v .
$$

The only sources of $\boldsymbol{H}$ considered here are the PM and the coil, which are both located inside the machine boundary. Thus, at the boundary of universe, the magnetic field $\boldsymbol{H}$ vanishes, and only the second term of (AI.4) remains

$$
i \lambda=\iint_{V_{\text {universe }}} \int \vec{B} \cdot \vec{H} d v \text {. }
$$

\section{APPENDIX II}

Equation (6) is once again written

$$
i\left(\lambda_{a}+\lambda_{\mathrm{PM}}\right)=\iint_{V_{\text {universe }}}\left(\vec{B}_{a}+\vec{B}_{\mathrm{PM}}\right) \cdot\left(\vec{H}_{a}+\vec{H}_{\mathrm{PM}}\right) d v .
$$

From Maxwell's equations, we have

$$
\begin{aligned}
\nabla \cdot \vec{B}_{\mathrm{PM}} & =0 \\
\nabla \cdot \vec{B}_{a} & =0
\end{aligned}
$$

and

$$
\nabla \times \vec{H}_{\mathrm{PM}}=0
$$

As demonstrated in [11], the integral taken over all space of the dot product of two vectors is zero, if the curl of the first vector is zero, and the divergence of the second vector is also zero. This identity is expressed as

$$
\begin{gathered}
\iiint_{V_{\text {universe }}} \vec{B}_{\mathrm{PM}} \cdot \vec{H}_{\mathrm{PM}} d v=0 \\
\iint_{V_{\text {universe }}} \int \vec{B}_{a} \cdot \vec{H}_{\mathrm{PM}} d v=0 .
\end{gathered}
$$

This allows us to rewrite (AII.1).

$$
i\left(\lambda_{a}+\lambda_{\mathrm{PM}}\right)=\iint_{V_{\text {universe }}}\left(\vec{B}_{a}+\vec{B}_{\mathrm{PM}}\right) \cdot \vec{H}_{a} d v
$$

This paper focuses on the flux linkage $\lambda_{\mathrm{PM}}$ created by the PM in the coil. To obtain $\lambda_{\mathrm{PM}}$, we can use (AII.5) and substract the component $i \lambda_{a}$. This is easily obtained from (5) by considering the case where the only fields and flux linkage present inside the machine are being created by the current flowing in the coil. We express $i \lambda_{a}$ as

$$
i \lambda_{a}=\iint_{V_{\text {universe }}} \int \vec{B}_{a} \cdot \vec{H}_{a} d v .
$$


Inserting (AII.6) into (AII.5), we obtain

$$
i \lambda_{\mathrm{PM}}=\iint_{V_{\text {universe }}} \int \vec{B}_{\mathrm{PM}} \cdot \vec{H}_{a} d v .
$$

This result is also obtained in [11]. In (AII.7), the domain of integration is still the entire universe. It is possible to simplify this expression further if we divide the total universe into four subdomains: the volume $V_{\mathrm{PM}}$ of the PM in the machine, the volume $V_{\text {iron }}$ of iron in the machine, the volume of air $V_{\text {air }}$ inside the machine, and the volume of all universe $V_{\text {remain }}$ outside the machine boundary. It must be noted that $V_{\text {air }}$ contains not only air, but all material for which permeability is unity (like coils, insulators, etc.). The four domains of integration are shown in Fig. 1.

\section{1) Subdomain $V_{\mathrm{PM}}$}

Inside the PM, the constitutive relation is given by (1).

We rewrite (AII.7) for the PM volume

$$
\begin{gathered}
\iint_{V_{\mathrm{PM}}} \int \vec{H}_{a} \cdot \vec{B}_{\mathrm{PM}} d v=\iint_{V_{\mathrm{PM}}} \mu_{0} \vec{H}_{a} \cdot \vec{H}_{\mathrm{PM}} d v \\
+\iint_{V_{\mathrm{PM}}} \int \vec{H}_{a} \cdot \vec{B}_{r} d v .
\end{gathered}
$$

Because $\boldsymbol{H}_{a}$ is the field obtained when the magnets are replaced by vacuum, we rewrite (AII.8)

$$
\begin{gathered}
\iint_{V_{\mathrm{PM}}} \int \vec{H}_{a} \cdot \vec{B}_{\mathrm{PM}} d v=\iint_{V_{\mathrm{PM}}} \int \vec{B}_{a} \cdot \vec{H}_{\mathrm{PM}} d v \\
+\iint_{V_{\mathrm{PM}}} \int \vec{H}_{a} \cdot \vec{B}_{r} d v
\end{gathered}
$$

2) Subdomain $V_{\text {iron }}$

Inside the iron, the magnetic fields $\boldsymbol{H}_{a}$ and $\boldsymbol{H}_{\mathrm{PM}}$ are zero, because of assumption 2 in Section II-A. We can write

$\iint_{V_{\text {iron }}} \int \vec{H}_{a} \cdot \vec{B}_{\mathrm{PM}} d v=\iint_{V_{\text {iron }}} \int \vec{B}_{a} \cdot \vec{H}_{\mathrm{PM}} d v=0$

3) Subdomain $V_{\text {air }}$

The subdomain of air also shows a reciprocity relation between $\boldsymbol{H}_{a}, \boldsymbol{B}_{\mathrm{PM}}$ and $\boldsymbol{B}_{a}, \boldsymbol{H}_{\mathrm{PM}}$. We can write

$$
\iint_{V_{\mathrm{Air}}} \int \vec{H}_{a} \cdot \vec{B}_{\mathrm{PM}} d v=\iint_{V_{\mathrm{Air}}} \int \vec{B}_{a} \cdot \vec{H}_{\mathrm{PM}} d v
$$

4) Subdomain $V_{\text {remain }}$

As defined previously, the vector quantities $\boldsymbol{H}_{a}, \boldsymbol{B}_{\mathrm{PM}}$, $\boldsymbol{B}_{a}$, and $\boldsymbol{H}_{\mathrm{PM}}$ are zero outside the machine boundary. We can write

$\iint_{\text {remain }} \int \vec{H}_{a} \cdot \vec{B}_{\mathrm{PM}} d v=\iint_{V_{\text {remain }}} \int \vec{B}_{a} \cdot \vec{H}_{\mathrm{PM}} d v=0$.
Summing the four subdomains, we obtain the entire universe. We insert (AII.9), (AII.10), (AII.11), and (AII.12) into (AII.7), and write

$$
i \lambda_{\mathrm{PM}}=\iint_{V_{\text {remain }}} \int \vec{B}_{a} \cdot \vec{H}_{\mathrm{PM}} d v+\iint_{V_{\mathrm{PM}}} \int \vec{H}_{a} \cdot \vec{B}_{r} d v
$$

Because of (AII.4), we can simplify (AII.13) to obtain

$$
i \lambda_{\mathrm{PM}}=\iint_{V_{\mathrm{PM}}} \int \vec{H}_{a} \cdot \vec{B}_{r} d v
$$

\section{APPENDIX III}

The conventional PM synchronous machine used throughout this paper has the following geometrical specifications:

Electrical time period $=20 \mathrm{~ms}$

Distance between two poles: $24 \mathrm{~mm}$

Distance between two slots: $8 \mathrm{~mm}$

Air gap: $2 \mathrm{~mm}$

Radial thickness of the PM: $6 \mathrm{~mm}$

Slot width: $4 \mathrm{~mm}$

Slot depth: $8 \mathrm{~mm}$

Material used for the PM:

$\mathrm{Nd}-\mathrm{Fe}-\mathrm{B}$

$\mathrm{B}_{\mathrm{r}}=1.23 \mathrm{~T}$ and $\mu_{\mathrm{rec}}=1.09$

Specifications for the rectangular PM shape (Fig. 1):

Width of the PM $=24 \mathrm{~mm}$

Distance between two adjacent PM $=0 \mathrm{~mm}$

Specifications for the V-shape PM (Fig. 6):

Width of the PM in the top part $=22 \mathrm{~mm}$

Width of the PM in the bottom part $=18 \mathrm{~mm}$

Distance between two adjacent PM $=2 \mathrm{~mm}$.

\section{REFERENCES}

[1] J. De La Ree and N. Boules, "Induced voltage harmonic reduction of PM cylindrical machines,” IEEE Trans. Ind. Applicat., vol. 28, pp. 619-624, June 1992.

[2] R. J. Strahan and D. B. Watson, "Effects of airgap and magnet shapes on permanent magnet reluctance torque," IEEE Trans. Magn., vol. 35, pp. 536-542, Jan. 1999.

[3] J. De La Ree and N. Boules, "Magnet shaping to reduce induced voltage harmonics in PM machines with surface mounted magnets," IEEE Trans. Energy Conversion, vol. 6, pp. 155-161, Mar. 1991.

[4] M. R. Dubois, H. Polinder, and J. A. Ferreira, "Varying magnetization orientation for permanent-magnet volume reduction in machines," IEEE Trans. Magn., vol. 39, pp. 1793-1799, May 2003.

[5] O. K. Mawardi, “On the concept of coenergy," J. Franklin Inst., vol. 264, no. 1 , pp. 313-332, 1957.

[6] J. A. Stratton, Electromagnetic Theory. New York: McGraw-Hill, 1941.

[7] E. Durand, Magnetostatic (in French). Paris, France: Masson, 1968.

[8] G. Bertotti, Hysteresis in Magnetism. New York: Academic, 1998.

[9] M. R. Harris, G. H. Pajooman, and S. M. Abu Sharkh, "Performance and design optimization of electric motors with heteropolar surface magnets and homopolar windings," Proc. Inst. Elect. Eng.—Electr. Power Appl., vol. 143, pp. 429-436, Nov. 1996.

[10] P. C. Matthews, Vector Calculus. New York: Springer-Verlag, 1998.

[11] W. F. Brown, Magnetostatic Principles in Ferromagnetism. Amsterdam, The Netherlands: North-Holland, 1962. 
Maxime R. Dubois (M'00) was born in Alma, QC, Canada, in 1968. $\mathrm{He}$ received the B.Ing. and M.Sc. degrees in electrical engineering from the Université Laval, Quebec, QC, in 1991 and 1993, respectively. He is working toward the Ph.D. degree at the Laboratory of Electrical Power Processing, Delft University of Technology, Delft, The Netherlands.

Between 1993 and 1999, he worked as a design engineer for private companies in Canada in the area of power electronics. Since 1999, he has been with the Laboratory of Electrical Power Processing, Delft University of Technology. His main interests are machines and power electronics applied to the generation of energy from renewable sources.

Henk Polinder (M'97) was born in Nunspeet, The Netherlands, in 1968. He received the M.Sc. degree in 1992 and the Ph.D. degree in 1998, both in electrical engineering, from Delft University of Technology, Delft, The Netherlands.

Currently, he is an Assistant Professor at the Electrical Power Processing Laboratory, Delft University of Technology, where he teaches courses on electrical machines and drives. His research interests are in the field of electromechanical power conversion. He currently works on direct drive generators for wind turbines, linear direct drive generators for wave energy, and actuators for highprecision motion control.
Jan A. Ferreira (M'88-SM'01) was born in Pretoria, South Africa. He received the B.Sc.Eng. (cum laude), M.Sc.Eng. (cum laude), and Ph.D. degrees in electrical engineering from the Rand Afrikaans University, Johannesburg, South Africa, in 1980, 1982, and 1988, respectively.

In 1981, he was with the Institute of Power Electronics and Electric Drives, Technical University of Aachen, Aachen, Germany, and worked in industry at ESD(Pty) Ltd from 1982 to 1985. From 1986 to 1997, he was on the Faculty of Engineering at Rand Afrikaans University, where he held the Carl and Emily Fuchs Chair of Power Electronics. Since 1998, he has been a Professor at the ITS Faculty of the Delft University of Technology, Delft, The Netherlands.

Prof. Ferreira is the Transactions Review Chairman of the IEEE IAS Power Electronic Devices and Components Committee, Vice-Chairman of the IEEE Joint IAS/PELS Benelux chapter, and Chairman of the CIGRE SC14 national committee of the Netherlands. He is also a member of the IEEE PESC Adcom and a member of the executive committee of the EPE Society. 